\title{
A!
}

This is an electronic reprint of the original article.

This reprint may differ from the original in pagination and typographic detail.

Johnston, Karen; Nieminen, Risto M.

\section{Polymer adhesion}

Published in:

Physical Review B

DOI:

10.1103/PhysRevB.76.085402

Published: 02/08/2007

Document Version

Publisher's PDF, also known as Version of record

Please cite the original version:

Johnston, K., \& Nieminen, R. M. (2007). Polymer adhesion: First-principles calculations of the adsorption of organic molecules onto Si surfaces. Physical Review B, 76(8), 1-8. [085402].

https://doi.org/10.1103/PhysRevB.76.085402

This material is protected by copyright and other intellectual property rights, and duplication or sale of all or part of any of the repository collections is not permitted, except that material may be duplicated by you for your research use or educational purposes in electronic or print form. You must obtain permission for any other use. Electronic or print copies may not be offered, whether for sale or otherwise to anyone who is not an authorised user. 


\title{
Polymer adhesion: First-principles calculations of the adsorption of organic molecules onto Si surfaces
}

\author{
Karen Johnston and Risto M. Nieminen \\ Laboratory of Physics, Helsinki University of Technology, P.O. Box 1100, 02015, Finland \\ (Received 6 February 2007; revised manuscript received 16 April 2007; published 2 August 2007)
}

\begin{abstract}
The structures and energetics of organic molecules adsorbed onto clean and H-passivated $\mathrm{Si}(001)-(2 \times 1)$ surfaces have been calculated using density functional theory. For benzene adsorbed on the clean Si surface the tight-bridge structure was found to be stable and the butterfly structure metastable. Both carbonic acid, $\mathrm{H}_{2} \mathrm{CO}_{3}$, and propane, $\mathrm{C}_{3} \mathrm{H}_{8}$, dissociate on contact with the surface. Passivation of the Si surface with $\mathrm{H}$ atoms has a dramatic effect on the surface properties. The passivated surface is very inert and the binding energy of all the molecules is very weak.
\end{abstract}

DOI: 10.1103/PhysRevB.76.085402

PACS number(s): 68.43.Fg, 68.43.Bc, 82.35.Gh

\section{INTRODUCTION}

The adsorption of organic molecules on semiconductor surfaces is of increasing importance to industry due to interest in the development of organic optoelectronic devices, molecular-scale electronics and biofunctionality. ${ }^{1-3}$ An additional motivation for studying these systems is to understand the nature of adhesion between plastics and metal or ceramic surfaces. Many of these materials do not adhere well and the main focus of this research is to obtain a better understanding of the structure and bonding at the polymer-surface interface.

While oxide and ceramic surfaces are primarily of interest, we have chosen to first study the simpler $\mathrm{Si}(001)$ surface, which is easier to simulate and will provide the initial insight into the nature of the bonding at surfaces. This will provide the basis for future work involving the more complex silica surface. The plastic of interest is mainly composed of the polymer bisphenol- $A$-polycarbonate (BPA-PC). The repeat unit, or monomer, is shown in Fig. 1. This polymer has been extensively studied with both first-principles methods ${ }^{4-6}$ and coarse-grained molecular dynamics simulations. ${ }^{7}$

To understand the nature of adhesion it is necessary to consider processes which occur at different time, length, and temperature scales and therefore a multiscale study is desirable. First-principles calculations provide details in the microscopic regime but they are too computationally demanding to be able to treat the entire polymer molecule on a surface. It is, therefore, necessary to divide the monomer into its analogous molecules (benzene, propane and carbonic acid as shown in Fig. 1) and study their individual adsorption on the surface. The results of these first-principles calculations could then be used to build potentials for coarse-grained simulations, so these calculations are the first step towards a multiscale study.

For low density systems, such as molecular adsorption, van der Waals forces can play a significant role. Recently a method for including the van der Waals energy within density functional theory (DFT) was suggested ${ }^{8,9}$ and has been shown to determine the correct binding distances and energies for benzene dimers ${ }^{10,11}$ and for benzene and naphthalene adsorbed on graphite. ${ }^{12,13}$ However, it is known that benzene binds strongly to $\mathrm{Si}$ and the additional binding due to van der
Waals forces will be small compared to the chemical binding energies. Thus, the effect of van der Waals forces has not been taken into account in this paper.

The paper is organized as follows: Section II describes the two $\mathrm{Si}$ surfaces under consideration and gives the technical details of the calculations. Sections III and IV describe the adsorption of benzene and the other organic molecules on the clean and H-passivated $\mathrm{Si}$ dimer surfaces, denoted $\mathrm{Si}(001)-(2 \times 1)$ and $\mathrm{Si}(001): \mathrm{H}-(2 \times 1)$, respectively. The discussion and conclusions appear in Sec. IV.

\section{II. $\mathrm{Si}(001)-(2 \times 1)$ AND $\mathrm{Si}(001): \mathrm{H}-(2 \times 1)$ SURFACES}

At room temperature the $\mathrm{Si}(001)$ surface has a $(2 \times 1)$ structure, which consists of the formation of buckled dimers, with one atom being drawn towards the surface closer to its three nearest neighbors, and the other being pushed away from its neighbors, as shown in Fig. 2. As the clean $\mathrm{Si}(001)-(2 \times 1)$ surface is rather reactive, adsorbed atoms or molecules, such as $\mathrm{H}$ or $\mathrm{H}_{2} \mathrm{O}$, are likely to be present and, therefore, we have also considered adsorption on the $\mathrm{H}$-passivated $\mathrm{Si}(001): \mathrm{H}-(2 \times 1)$ surface. The $\mathrm{H}-(2 \times 1)$ surface is passivated by one $\mathrm{H}$ atom per $\mathrm{Si}$ atom, as shown in Fig. 2.



FIG. 1. (Color online) The monomer bisphenol-A-polycarbonate (BPA-PC) and its analogous molecules, benzene $\left(\mathrm{C}_{6} \mathrm{H}_{6}\right)$, carbonic acid $\left(\mathrm{H}_{2} \mathrm{CO}_{3}\right)$, benzene and propane $\left(\mathrm{C}_{3} \mathrm{H}_{8}\right)$. 

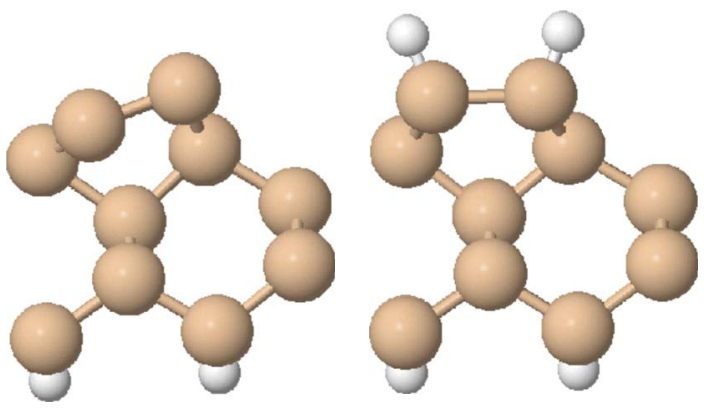

FIG. 2. (Color online) The clean $\mathrm{Si}(001)-(2 \times 1)$ dimer reconstruction (left) and H-passivated $\mathrm{Si}(001): \mathrm{H}-(2 \times 1)$ surface (right) viewed along the [110] direction.

First-principles calculations were performed with the Vienna $A b$ initio Simulation Package (VASP), ${ }^{14,15}$ which implements density functional theory. The PW91 flavor ${ }^{16,17}$ of the generalized-gradient approximation (GGA) and projector augmented-wave potentials ${ }^{18}$ were used. The plane-wave energy cutoff was $400 \mathrm{eV}$, which corresponds to an error in the total energy of $\approx 3 \mathrm{meV}$ per Si atom, and the Brillouin zone mesh was equivalent to a $4 \times 4 \times 1$ Monkhorst-Pack $k$-point sampling for a $0.5 \mathrm{ML}$ supercell with dimensions $\left(\begin{array}{lll}1 & 1 & 0\end{array}\right)\left(\begin{array}{lllll}-1 & 1 & 0\end{array}\right)\left(\begin{array}{lll}0 & 0 & 4\end{array}\right)$. The calculated lattice constant of bulk $\mathrm{Si}$ is $5.47 \AA$.

For the slab calculations we used a 5-atomic layer Si slab and $\approx 15 \AA$ of vacuum. All relaxations were considered complete when the forces were less than $10 \mathrm{meV} \AA^{-1}$. To save computational time only adsorption on one side of the slab was considered. When the slab is asymmetric and periodic boundary conditions are used, as is the case in these calculations, it must be ensured that there is no dipole-dipole interaction between the slabs. The energy of the dipole-dipole interaction is inversely proportional to the distance between the slabs so one way to avoid this interaction is to have a large vacuum region between slabs. To check this the vacuum was increased from $\approx 15 \AA$ to $\approx 25 \AA$ and it was found that the adsorption energies did not change. This indicates that the dipole-dipole interaction is negligible. For both the clean and H-passivated slabs the bottom layer of the slab was fixed in the bulk Si positions and passivated with two $\mathrm{H}$ atoms per $\mathrm{Si}$ atom. The $\mathrm{Si}(001)-(2 \times 1)$ surface structure of a nine-atomic-layer slab was also calculated and the differences between the nine- and five-atomic-layer slabs were small, i.e., the dimer bond lengths are $2.31 \AA$ and $2.30 \AA$ and the angles are $18.11^{\circ}$ and $18.02^{\circ}$, respectively. Increasing the
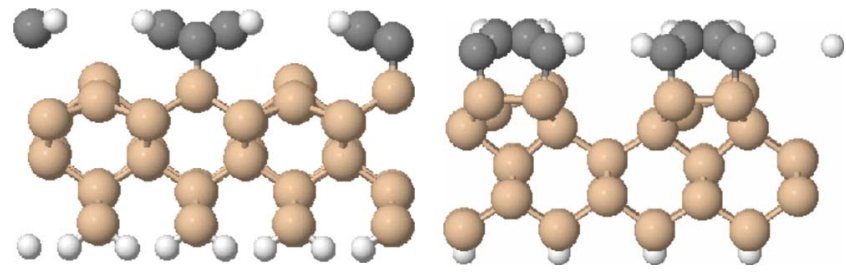

FIG. 3. (Color online) "Butterfly" structure of $\mathrm{C}_{6} \mathrm{H}_{6}$ on $\mathrm{Si}(001)-(2 \times 1)$ viewed along [110] and [110] (dimer rows), respectively.
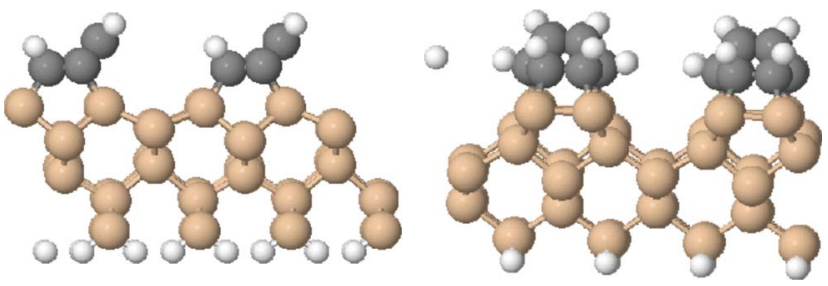

FIG. 4. (Color online) "Tight-bridge" structure of $\mathrm{C}_{6} \mathrm{H}_{6}$ on $\mathrm{Si}(001)-(2 \times 1)$ viewed along [110] and [110], respectively.

plane-wave cutoff energy to $600 \mathrm{eV}$ does not change these values. To check that the chemisorption energies are converged we also calculated the benzene adsorption energies on a nine-atomic-layer slab (see Sec. III A). The results presented in this paper use H-passivated five-atomic-layer slabs and a cutoff of $400 \mathrm{eV}$ unless otherwise indicated. For the $\mathrm{Si}(001): \mathrm{H}-(2 \times 1)$ surface the H-passivation caused the dimer to flatten and the bond length to increase to $2.42 \AA$, in agreement with Zanella et al. ${ }^{19}$

\section{ADSORPTION ON Si(001)- $(2 \times 1)$}

\section{A. Benzene, $\mathrm{C}_{6} \mathrm{H}_{6}$}

There are several publications which report the structural geometry and energetics of benzene adsorbed on $\mathrm{Si}(001)-(2 \times 1)$. Five different geometries have been studied in the literature: two single dimer di- $\sigma$ bonded structures named "tilted" and "butterfly," and three double dimer tetra$\sigma$ bonded structures: "tight bridge," "twisted bridge," and the symmetric bridge ("pedestal"). The two most stable structures are agreed to be the butterfly and tight-bridge structures but to date there is no conclusive experimental or computational study that determines which structure is the stable one and which is metastable. Here we make a comprehensive overview of the available data and present results for benzene adsorption at low coverages.

\section{Structural data}

Figures 3 and 4 show the butterfly and tight-bridge structures, respectively. The butterfly structure is bonded to the two dangling bonds of a single $\mathrm{Si}-\mathrm{Si}$ dimer whereas the tight-bridge structure is bonded to two $\mathrm{Si}-\mathrm{Si}$ dimers. These adsorption structures are significantly distorted compared to the isolated benzene molecule. Another distinguishing feature is that the butterfly structure has a symmetry plane along the Si dimer.

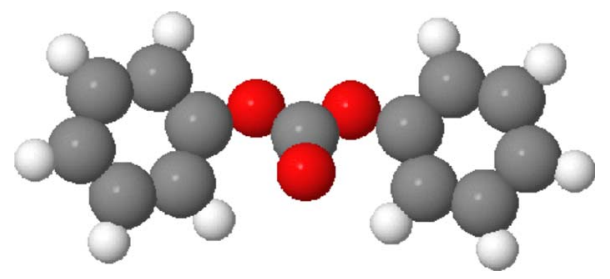

FIG. 5. (Color online) The $\mathrm{C}_{6} \mathrm{H}_{5} \mathrm{CO}_{3} \mathrm{C}_{6} \mathrm{H}_{5}$ molecule represents part of the BPA-PC monomer containing the carbonate group. 
TABLE I. Bond lengths $(\AA)$ for the butterfly geometry. $\mathrm{Si}_{\mathrm{C}}$ and $\mathrm{C}_{\mathrm{Si}}$ denote $\mathrm{Si}$ atoms bonded to $\mathrm{C}$ and $\mathrm{C}$ atoms bonded to $\mathrm{Si}$, respectively.

\begin{tabular}{lccccc}
\hline \hline $\mathrm{Si}-\mathrm{Si}$ & $\mathrm{Si}_{\mathrm{C}}-\mathrm{Si}_{\mathrm{C}}$ & $\mathrm{Si}_{\mathrm{C}}-\mathrm{C}_{\mathrm{Si}}$ & $\mathrm{C}-\mathrm{C}_{\mathrm{Si}}$ & $\mathrm{C}=\mathrm{C}$ & Reference \\
\hline 2.34 & 2.39 & 1.98 & 1.50 & 1.35 & Present \\
2.24 & 2.34 & 1.98 & 1.51 & 1.34 & 21 \\
& 2.39 & 1.89 & $1.47-1.49$ & 1.35 & 20 \\
& & 1.97 & 1.51 & & 22 \\
\hline \hline
\end{tabular}

Our structural data for both geometries is in excellent agreement with previous first-principles results (see Tables I and II). In the butterfly structure the benzene adopts a "V" shape as shown in Fig. 3. The $\mathrm{Si}$ dimer bond length is $2.39 \AA$, which is in excellent agreement with Hofer et al. ${ }^{20}$

\section{Stability-experimental evidence}

According to a scanning tunneling microscopy (STM) study by Lopinski et al., ${ }^{24}$ the benzene molecule adsorbs initially in the butterfly structure but this is observed to be metastable with respect to a bridging configuration. They were able to convert the benzene from one structure to another using the STM tip and estimated the conversion barrier to be $0.95 \mathrm{eV}$.

Gokhale et $a .^{25}$ used thermal desorption spectroscopy (TPD) and angle-resolved photoelectron spectroscopy (ARUPS) to investigate the electronic structure and symmetry of benzene on $\mathrm{Si}$ and observed a single dimer structure, supporting the butterfly configuration.

Witkowski et al. $^{26}$ used near-edge x-ray-absoprtion fine structure (NEXAFS) to look at the structure of the adsorbed benzene and found the benzene to be symmetric with respect to the dimer axis, ruling out the tight-bridge structure. These findings were supported by reflectance anisotropy spectroscopy (RAS) and surface differential reflectivity spectroscopy (SDRS) data, ${ }^{27}$ which found that benzene adsorbs on top of a single dimer rather than on the bridge site between two dimers.

The high-resolution photoemission study by Kim et al. ${ }^{28}$ suggested that the adsorption geometry depends on the coverage. ${ }^{37}$ They found that at low coverages a bridging structure is favored but that at high coverages a single dimer structure is more stable. This result is consistent with the previous experiments as the STM measurements were carried out at a low coverage and the TPD, ARUPS, NEXAFS, RAS, and SDRS experiments were carried out at the saturation coverage of $0.5 \mathrm{ML}^{25,27,28}$

\section{Adsorption energies}

DFT studies of benzene adsorbed on silicon all agree that the tight-bridge structure is stable with the butterfly structure being metastable..$^{20,29,30}$ Lee et al. ${ }^{29}$ studied the tight-bridge and butterfly states using VASP and US/norm-conserving (NC) pseudopotentials. For a coverage of $0.5 \mathrm{ML}$ the tightbridge structure was more stable with an adsorption energy of $1.05 \mathrm{eV}$. The adsorption energy of the butterfly structure was $0.82 \mathrm{eV}$. They also studied the conversion between the butterfly and tight-bridge states and found a high-energy barrier of $0.87 \mathrm{eV}$, which means that both states could coexist. This supports the STM findings ${ }^{24}$ but disagrees with other experiments. A comparison between the present adsorption energies and those of previous studies is shown in Table III.

In the calculations shown in Table III the adsorption energies cover a rather large range, which could be due to different pseudopotentials or supercell size. All the results used the PW91 or PBE (Refs. 31-33) GGA's, which should give similar results. The current calculations are the most accurate calculations to date and we have tested the effect of using different slab thicknesses and pseudopotentials. Although these make some small changes to the adsorption energies they do not explain the large variation seen in the literature and hence we must attribute the differences to other convergence parameters.

\section{Coverage dependence}

Molecular coverage can also have a significant effect on adsorption as demonstrated by Kim et al., ${ }^{28}$ who observed that the structure of the adsorbed benzene is coverage dependent, with the butterfly structure stable at high coverages. Results for a range of coverages are presented in Tables IV and V. For the $0.25 \mathrm{ML}$ coverages two supercell orientations are possible. Supercells (a) and (b) have primitive lattice


tively, with the dimer rows along the $[\overline{1} 10]$ direction. Super-

TABLE II. Bond lengths ( $)$ for tight-bridge geometry.

\begin{tabular}{lccccc}
\hline \hline $\mathrm{Si}_{\mathrm{C}}-\mathrm{Si}_{\mathrm{C}}$ & $\mathrm{Si}_{\mathrm{C}}-\mathrm{C}_{\mathrm{Si}}$ & $\mathrm{C}_{\mathrm{Si}}-\mathrm{C}_{\mathrm{Si}}$ & $\mathrm{C}-\mathrm{C}_{\mathrm{Si}}$ & $\mathrm{C}=\mathrm{C}$ & Reference \\
\hline $2.35-2.38$ & $1.99-2.00$ & $1.57-1.58$ & 1.50 & 1.35 & Present \\
2.34 & $1.98-2.00$ & $1.58-1.59$ & 1.51 & 1.34 & 21 \\
2.39 & $1.98-2.01$ & 1.57 & 1.50 & 1.35 & 20 \\
& $1.87-1.89$ & $1.47-1.53$ & & 1.35 & 22 \\
\hline \hline
\end{tabular}


TABLE III. Adsorption energies of benzene in the tight-bridge (TB) and butterfly (BF) geometries for a coverage of $0.5 \mathrm{ML}$.

\begin{tabular}{|c|c|c|c|}
\hline \multicolumn{2}{|c|}{$\begin{array}{l}\text { Adsorption energy } \\
\left(\mathrm{eV} \text { molecule }{ }^{-1}\right)\end{array}$} & \multirow[b]{2}{*}{ Details $^{\mathrm{a}}$} & \multirow[b]{2}{*}{ Reference } \\
\hline TB & $\mathrm{BF}$ & & \\
\hline 1.21 & 0.99 & 9, $400 \mathrm{eV}, \mathrm{US}$ & Present \\
\hline 1.26 & 1.01 & 9, $600 \mathrm{eV}$, PAW & Present \\
\hline 1.25 & 1.00 & 9, $400 \mathrm{eV}$, PAW & Present \\
\hline 1.21 & 1.02 & 5, $400 \mathrm{eV}$, PAW & Present \\
\hline 0.98 & 0.88 & $6,350 \mathrm{eV}, \mathrm{US}$ & 30 \\
\hline 1.05 & 0.82 & $5,340 \mathrm{eV}, \mathrm{US} / \mathrm{NC}$ & 29 \\
\hline 1.18 & & 8, $300 \mathrm{eV}, \mathrm{US}$ & 20 \\
\hline
\end{tabular}

The details are as follows: atomic layers of $\mathrm{Si}$ in slab, plane-wave cutoff energy, pseudopotential type $(\mathrm{US}=$ ultrasoft, $\mathrm{PAW}=$ projector augmented wave, $\mathrm{NC}=$ norm-conserving). All the calculations used either the PW91 or PBE GGAs, which are similar.

cell (b) has four dimers along the dimer row.

The butterfly structure was found to be unstable at a coverage of $1 \mathrm{ML}$, which is consistent with the experimental saturation coverage of around $0.5 \mathrm{ML} .^{25,27,28}$ To obtain a 1 ML coverage for the tight-bridge structure the $\mathrm{Si}$ dimers would have to be shared between the benzene molecules and, thus, for this case a coverage of $1 \mathrm{ML}$ is unrealistic.

The present results show a small variation in adsorption energies for coverages of $0.125-0.5 \mathrm{ML}$ and for all coverages the tight-bridge structure remains stable. The adsorption energies for the butterfly structure do not change significantly when the slab is increased from five to nine layers. However for the tight-bridge structure additional relaxation in the nine-layer slab results in higher adsorption energies, particularly for the 0.25 (b) and 0.125 ML coverages.

Hofer et $a .^{20}$ found that increasing the coverage from 0.25 ML to 0.5 ML decreased the binding energy of the tight-bridge structure by $0.24 \mathrm{eV}$, which was attributed to a relaxation of the strain within the cell. This agrees qualitatively with the present results, which show a smaller decrease in the tight-bridge adsorption energy of $0.1 \mathrm{eV}$. These results disagree with the DFT results of Lee et al. ${ }^{29}$ who observed an increase of $0.14 \mathrm{eV}$.
There are two possible scenarios which could explain the conflicting experimental and theoretical evidence. The first possibility is that the benzene molecule adsorbs initially in the butterfly structure ${ }^{24}$ (there is no barrier for this reaction as shown in Fig. 11) but this is metastable with respect to a bridging configuration. The conversion barrier was estimated to be $0.95 \mathrm{eV}$, which is in good agreement with the DFT calculations of Lee et al., ${ }^{29}$ who found the conversion barrier to be $0.87 \mathrm{eV}$. The barrier is high enough to allow the butterfly configuration to exist for a relatively long time, which could explain why other experimental methods have observed the butterfly structure.

The second possibility is that the inclusion of van der Waals forces will affect the stabilities. One of the drawbacks of density functional theory is that it does not include van der Waals forces, which are due to dynamical correlation. Even though benzene binds strongly to $\mathrm{Si}$ and the additional binding due to van der Waals forces will be small compared to the chemical binding energies, the van der Waals forces may be large enough to overcome the energy differences between the two structures and stabilize the butterfly structure. The quantum mechanics/molecular mechanics method used by Jung et al. ${ }^{21}$ included van der Waals forces by using singlepoint energy calculations with multireference second-order perturbation theory. They found that for an isolated molecule on a $\mathrm{Si}$ cluster the butterfly structure was more stable than the tight-bridge structure, with adsorption energies of 1.04 and $0.96 \mathrm{eV}$, respectively. However, they used a small $\mathrm{Si}$ cluster, which may not be large enough to represent the $\mathrm{Si}$ surface accurately, so it is not clear whether this result is valid. Furthermore, the cluster geometry corresponds to an isolated molecule, i.e., a low coverage system, which according to Kim et al. is in the regime where the tight-bridge structure should be stable.

\section{B. Carbonic acid, $\mathrm{H}_{2} \mathrm{CO}_{3}$}

When carbonic acid is placed on the clean Si surface it dissociates with one $\mathrm{O}$ and two $\mathrm{H}$ atoms bonding to the $\mathrm{Si}$ surface and a $\mathrm{CO}_{2}$ molecule being left over. The energy vs. distance of the $\mathrm{H}_{2} \mathrm{CO}_{3}$ approaching the surface was obtained by fixing the distance of the $\mathrm{C}$ atom from the surface and relaxing the molecule and surface. This shows that there is

TABLE IV. Variation of adsorption energy with coverage for the tight-bridge structure.

\begin{tabular}{|c|c|c|c|c|c|}
\hline \multirow{3}{*}{$\begin{array}{l}\text { Coverage } \\
(\mathrm{ML})\end{array}$} & \multicolumn{5}{|c|}{ Adsorption energy $(\mathrm{eV})$} \\
\hline & \multicolumn{2}{|c|}{ Present } & \multirow[b]{2}{*}{ Lee (Ref. 29) } & \multirow[b]{2}{*}{ Hofer (Ref. 20) } & \multirow[b]{2}{*}{ Jung (Ref. 21) } \\
\hline & five layer & nine layer & & & \\
\hline Isolated & & & & & 0.96 \\
\hline 0.125 & 1.25 & 1.34 & & & \\
\hline 0.25 (a) & 1.23 & 1.27 & & & \\
\hline $0.25(b)$ & 1.24 & 1.35 & 0.91 & $1.42^{\mathrm{a}}$ & \\
\hline 0.5 & 1.21 & 1.25 & 1.05 & 1.18 & \\
\hline
\end{tabular}

The supercell lattice vectors for the 0.25 ML case are not stated in Ref. 20. 



FIG. 6. (Color online) The lowest energy adsorption structure of $\mathrm{C}_{6} \mathrm{H}_{5} \mathrm{CO}_{3} \mathrm{C}_{6} \mathrm{H}_{5}$ adsorbed on $\mathrm{Si}$. Both phenol rings have the tightbridge structure. Left: viewed along [110]. Right: viewed along [110] dimer rows.

no energy barrier to overcome to enable this reaction to occur.

To avoid the problem of dissociation it is necessary to consider the possible conformations of carbonic acid within the BPA-PC chain. The structure of this section of BPA-PC is shown in Fig. 5.

Based on our knowledge of benzene adsorption on Si the most stable structure is likely to have the two phenol rings adsorbed in the tight-bridge structure, as shown in Fig. 6. The molecule is arranged so that the $\mathrm{CO}_{3}$ group is attached to the top of the two tight-bridge structures. Several orientations of the $\mathrm{CO}_{3}$ group within the adsorbed $\mathrm{C}_{6} \mathrm{H}_{5} \mathrm{CO}_{3} \mathrm{C}_{6} \mathrm{H}_{5}$ molecule were calculated and the orientation with the minimum energy has the $\mathrm{C}=\mathrm{O}$ double bond pointing upwards and inwards, as shown in Fig. 6. The adsorption energy of the whole molecule is $1.83 \mathrm{eV}$. By subtracting the adsorption energy of the two tight-bridge structures from this adsorption energy we estimated the adsorption energy of the $\mathrm{CO}_{3}$ group to be $-0.59 \mathrm{eV}$. This means that the $\mathrm{CO}_{3}$ experiences a repulsive force from the Si surface.

Other adsorption configurations for this molecule are unlikely as the $\mathrm{CO}_{3}$ cannot stretch from a tight bridge to a butterfly configuration or from the high point of one tightbridge to the low point of an adjacent tight-bridge. However, the adsorption barrier for this structure may be high since due to the geometrical constraints it may be difficult for both benzenes to initially adsorb in the butterfly structure.

\section{Propane, $\mathrm{C}_{3} \mathrm{H}_{8}$}

Similar to the case of carbonic acid, the propane molecule dissociates on the surface, with the two $\mathrm{H}$ atoms bonding to

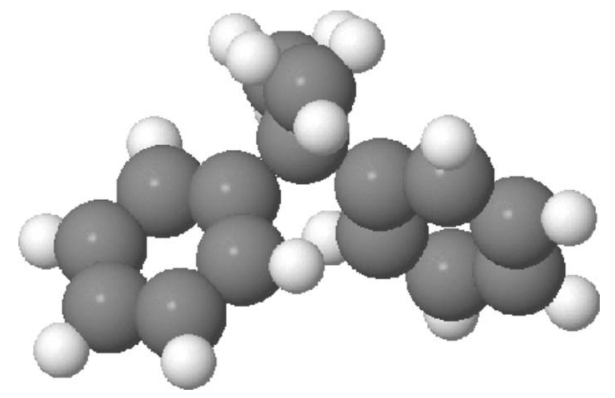

FIG. 7. The isolated $\mathrm{C}_{6} \mathrm{H}_{5} \mathrm{C}_{3} \mathrm{H}_{6} \mathrm{C}_{6} \mathrm{H}_{5}$ molecule which is a segment of BPA-PC containing the propane group.
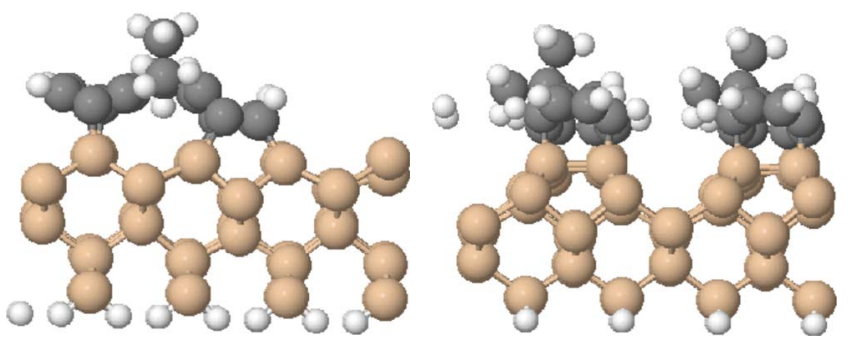

FIG. 8. (Color online) $\mathrm{C}_{6} \mathrm{H}_{5} \mathrm{C}_{3} \mathrm{H}_{6} \mathrm{C}_{6} \mathrm{H}_{5}$ on $\mathrm{Si}(001)-(2 \times 1)$ viewed along [110] and [110], respectively.

the surface. As before, we need to consider a larger segment of BPA-PC which contains the propane group.

The isolated molecule is shown in Fig. 7 and the molecule adsorbed on the Si surface is shown in Fig. 8. Geometrically the propane group cannot bond to two tight-bridge structures but it may bond to a tight bridge on the one side and the butterfly geometry on the other side. The lowest energy structure for this configuration is shown in Fig. 8 and the adsorption energy of the whole molecule is $1.59 \mathrm{eV}$. The estimated adsorption energy of the $\mathrm{C}_{3} \mathrm{H}_{6}$ group is $-0.64 \mathrm{eV}$, which corresponds to a repulsive force from the surface.

\section{ADSORPTION ON Si(001):H- $(2 \times 1)$}

The clean Si surface is very reactive so it is interesting to know how this compares to the H-passivated surface. Six adsorption sites have been considered and are shown in Fig. 9. Sites $A, B, C$, and $D$ are high symmetry positions, site $F$ is above a surface $\mathrm{H}$ atom, and site $E$ is the midpoint between two surface $\mathrm{H}$ atoms.

\section{A. Benzene, $\mathrm{C}_{6} \mathrm{H}_{6}$}

There are two orientations for the benzene molecule, denoted (I) and (II). Orientation (I) has two of the $\mathrm{C}-\mathrm{C}$ bonds in the benzene ring perpendicular to the $\mathrm{Si}-\mathrm{Si}$ dimers and orientation (II) is obtained by rotating (I) by $30^{\circ}$ around the vertical axis. As an example, benzene placed on site $C$ with orientation (II), is shown in Fig. 10.

For all sites and orientations the adsorption of benzene is very weak, with adsorption energies of $0.06-0.07 \mathrm{eV}$ and

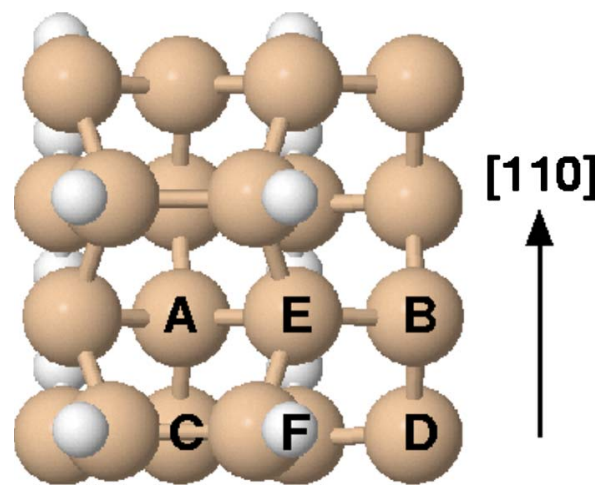

FIG. 9. (Color online) Adsorption sites on $\mathrm{Si}(001): \mathrm{H}-(2 \times 1)$. 

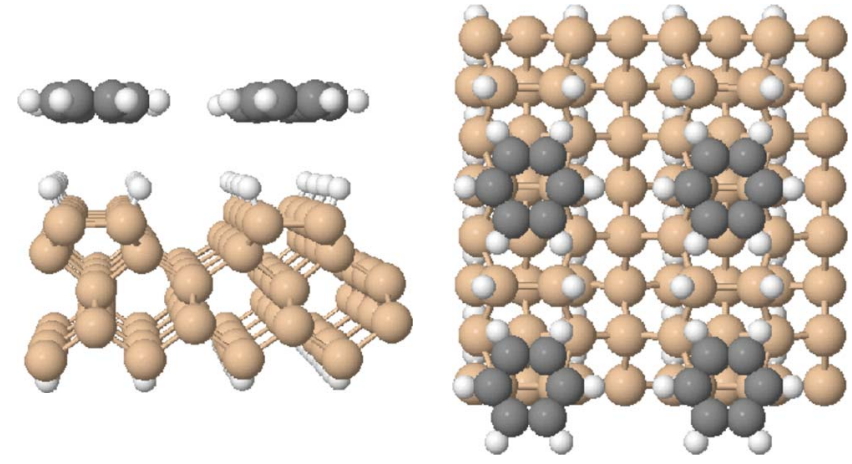

FIG. 10. (Color online) Side and top views of benzene adsorbed on site $C$ of the $\mathrm{Si}(001): \mathrm{H}-(2 \times 1)$ surface and with orientation (II).

equilibrium distances between the benzene and the surface $\mathrm{H}$ atoms of 3.2-3.4 $\AA$. The benzene molecule remains flat in contrast to the large distortion seen in the case on the clean Si surface.

The difference between the adsorption behavior of benzene on the clean $\mathrm{Si}$ surface and its behavior on the passivated surface is further demonstrated by looking at the variation of the adsorption energy as a function of distance from the surface, which is shown in Fig. 11. On the clean Si surface it is known that benzene initially adsorbs in the butterfly configuration, so for convenience the benzene was placed in configuration C(II) on each surface. For a particular separation distance the position of one of the $\mathrm{C}$ atoms was fixed and the rest of the benzene molecule and the surface were allowed to relax. For the clean surface it was convenient to fix either of the $\mathrm{C}$ atoms above the dimer $\mathrm{Si}$ atoms and the distance was defined as the difference between the $z$ position of the fixed carbon atom and the average $z$ position of the two $\mathrm{Si}$ dimer atoms in the minimum energy configuration. For the passivated surface the distance was defined as the distance from the fixed carbon atom to the $z$ position of the surface $\mathrm{H}$ atoms at the minimum energy configuration. The position of the $\mathrm{H}$ atoms is not significantly different for the various benzene configurations. As can be seen from Fig. 11 there is no barrier for adsorption into the butterfly configuration on the clean Si surface. For the H-passivated surface, the benzene experiences significant repulsion as it ap-

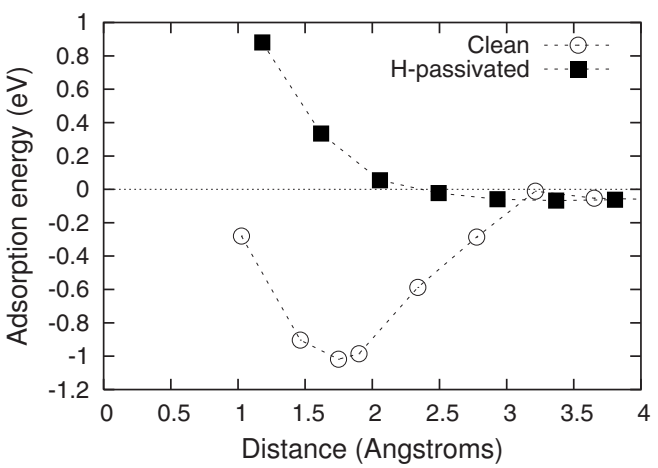

FIG. 11. Barriers for adsorption of $\mathrm{C}_{6} \mathrm{H}_{6}$ on site $\mathrm{C}(\mathrm{II})$ of $\mathrm{Si}(001)-(2 \times 1)$ and $\mathrm{Si}(001): \mathrm{H}-(2 \times 1)$.

proaches the surface and thus the benzene sees the surface as a approximately uniform hard wall.

The binding energies for the passivated surface are small and therefore to get the true adsorption energies and equilibrium distances of these molecules it is necessary to consider van der Waals interactions. Based on calculations of phenol on alumina ${ }^{12}$ and phenol on graphite ${ }^{13}$ we estimate the van der Waals energy to be of the order of $0.2-0.5 \mathrm{eV}$.

\section{B. Carbonic acid, $\mathrm{H}_{2} \mathrm{CO}_{3}$}

There are four possible orientations for the $\mathrm{H}_{2} \mathrm{CO}_{3}$ molecule to adsorb on the H-passivated $\mathrm{Si}(001)$ surface. These are (I) parallel to a dimer with the double-bonded $\mathrm{O}$ on top, (II) perpendicular to a dimer with the double-bonded $\mathrm{O}$ on top, (III) parallel with the double-bonded $\mathrm{O}$ pointing down and (IV) perpendicular with the double-bonded $\mathrm{O}$ pointing down. Configuration B(III) is shown in Fig. 12.

The binding energies for all the sites are in the range 0.04-0.10 eV and the equilibrium distances (measured from the $\mathrm{C}$ atom in $\mathrm{H}_{2} \mathrm{CO}_{3}$ to the surface $\mathrm{H}$ atoms) are in the range 1.6-4.2 eV. The maximum binding energy and minimum adsorption distance occurs for configuration $\mathrm{B}$ (III) and as can be seen from Fig. 12 this is mainly due to topology.

\section{Propane, $\mathrm{C}_{3} \mathrm{H}_{8}$}

There are four possible orientations in which propane could adsorb. In (I) and (III) the carbon chain is parallel to

TABLE V. Variation of adsorption energy with coverage for the butterfly structure.

\begin{tabular}{|c|c|c|c|c|c|}
\hline \multirow{3}{*}{$\begin{array}{l}\text { Coverage } \\
\text { (ML) }\end{array}$} & \multicolumn{5}{|c|}{ Adsorption energy $(\mathrm{eV})$} \\
\hline & \multicolumn{2}{|c|}{ Present } & \multirow[b]{2}{*}{ Lee (Ref. 29) } & \multirow[b]{2}{*}{ Hofer (Ref. 20) } & \multirow[b]{2}{*}{ Jung (Ref. 21) } \\
\hline & five layer & nine layer & & & \\
\hline Isolated & & & & & 1.04 \\
\hline 0.125 & 1.07 & 1.06 & & & \\
\hline 0.25 (a) & 1.04 & 1.03 & & & \\
\hline $0.25(b)$ & 1.05 & 1.04 & 0.84 & $1.12^{\mathrm{a}}$ & \\
\hline 0.5 & 1.02 & 1.00 & 0.82 & & \\
\hline
\end{tabular}

The supercell lattice vectors for the 0.25 ML case are not stated in Ref. 20. 

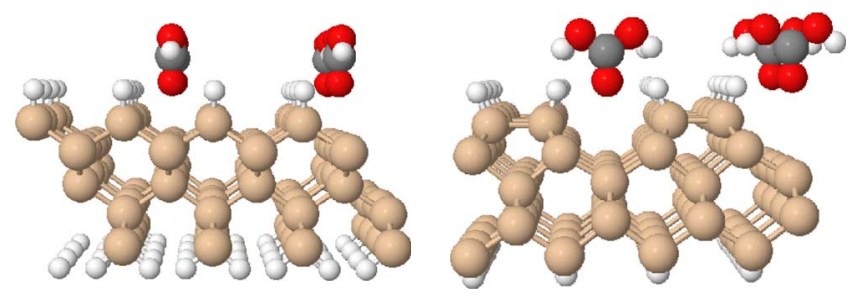

FIG. 12. (Color online) Carbonic acid molecule adsorbed on the $\mathrm{H}$-passivated $\mathrm{Si}$ surface with configuration B(III). Left: viewed along [110]. Right: viewed along [110] dimer rows.

the dimers, whereas in (II) and (IV) the carbon chain is perpendicular to the dimers. For (I) and (II) the carbon chain makes a "V" shape on the surface and (III) and (IV) the carbon chain is inverted to make an upside down "V." Figure 13 shows the propane molecule in site $C$ and orientation (I), in which the "V" shape can be seen on the right-hand side. Similar to benzene and carbonic acid, propane is also very weakly bound to the surface with adsorption energies ranging from $0.04-0.06 \mathrm{eV}$. For orientations (I) and (II) the vertical distance from the central $\mathrm{C}$ in propane to the surface $\mathrm{H}$-atoms is $2.5-3.4 \AA$ and for orientations (III) and (IV) it is $3.8-4.2 \AA$.

\section{SUMMARY AND DISCUSSION}

The adsorption behavior of benzene, carbonic acid, and propane on the $\mathrm{Si}(001)-(2 \times 1)$ and $\mathrm{Si}(001): \mathrm{H}-(2 \times 1)$ surfaces was calculated. For the $\operatorname{Si}(001)-(2 \times 1)$ surface the stable adsorption structure of benzene was found to be the tight-bridge structure with an adsorption energy of $1.26 \mathrm{eV}$. The butterfly structure is metastable with an adsorption energy of $1.01 \mathrm{eV}$. The carbonic acid and propane molecules were found to spontaneously dissociate on the surface.

To avoid the issue of dissociation, the adsorption behavior of larger segments of the BPA-PC chain was studied. These segments correspond to carbonic acid bonded to two benzene rings and propane bonded to two benzene rings. Trial struc-
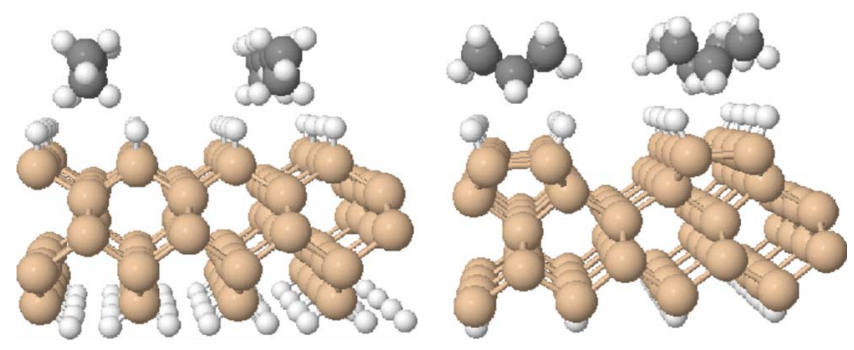

FIG. 13. (Color online) Propane molecule adsorbed on the H-passivated Si surface with configuration (I). Left: viewed along [1]10]. Right: viewed along [110] dimer rows. tures of these large molecules were calculated and the results were used to estimate adsorption energies of the $\mathrm{CO}_{3}$ and $\mathrm{C}_{3} \mathrm{H}_{6}$ groups. It was found that these groups are repelled from the Si surface.

Combined DFT and coarse-grained studies of BPA-PC on $\mathrm{Ni}(111)$ have already been published. ${ }^{34,35}$ The first-principles calculations showed that benzene binds strongly to the $\mathrm{Ni}$ surface but that carbonic acid and propane experience significant repulsion. The behavior of these molecules on $\mathrm{Si}$ is very different to their behavior on $\mathrm{Ni}(111)$. The adsorption energy of benzene on $\mathrm{Ni}$ is $1.05 \mathrm{eV},{ }^{34}$ which is comparable to the adsorption energy of benzene on $\mathrm{Si}$. The geometries, however, are very different, and on the Ni surface the benzene remains relatively undistorted at a center-of-mass distance of $2 \AA$. In contrast, propane and carbonic acid see the $\mathrm{Ni}$ surface as a uniform hard wall and they experience significant repulsion for distances shorter than $3.2 \AA$. By incorporating this data into a coarse-grained model, they showed that the phenol chain ends bind to the Ni surface and that the rest of the polymer is repelled away from the surface.

An important point to note is that the trial adsorption structures for the BPA-PC segments, described above, may not be geometrically compatible with the larger BPA-PC chain. The polymer chain must be continued from the low point of the tight-bridge structure and if the repulsion of the $\mathrm{C}_{3} \mathrm{H}_{6}$ group is strong enough this benzene may desorb from the surface. In this case the "internal" benzene groups may not approach the surface and only the benzene chain ends may adsorb. If this is the case, then despite the differences in molecular adsorption behavior the behavior of a BPA-PC chain on Si may turn out to be similar to that of BPA-PC on Ni.

Passivation of the Si surface with $\mathrm{H}$ atoms has a dramatic effect on the surface properties. The passivated surface is very inert and the binding energy of all the molecules is extremely weak. Due to the weak binding of this system the van der Waals interaction becomes dominant and to get a true picture of the binding in this case it would be necessary to include the van der Waals energy.

Another physical effect that is missing in the DFT formalism is the image charge potential. This has been addressed in a recent study of adsorbed molecules on graphite. ${ }^{36}$ The image charge affects, in particular, the excited-state energy levels but not the adsorption energies.

This work provides the first step in multiscale simulations of the adhesion of polymers to surfaces. The first-principles calculations presented in this paper can now be used to build potentials for coarse-grained simulations.

\section{ACKNOWLEDGMENTS}

The authors would like to thank Janne Blomqvist, Petri Salo, Jyrki Vuorinen, and Luigi Delle Site for useful discussions. Support was provided by the Finnish Funding Agency for Technology and Innovation (TEKES). Computational resources were provided by the Finnish IT Center for Science. 
${ }^{1}$ G. Lopinski, D. Wayner, and R. Wolkow, Nature (London) 406, 48 (2000).

${ }^{2}$ N. A. Besley and A. J. Blundy, J. Phys. Chem. B 110, 1701 (2006).

${ }^{3}$ S. Y. Quek, J. B. Neaton, M. S. Hybertsen, E. Kaxiras, and S. G. Louie, Phys. Status Solidi B 243, 2048 (2006).

${ }^{4}$ B. Montanari, P. Ballone, and R. Jones, J. Chem. Phys. 108, 6947 (1998).

${ }^{5}$ B. Montanari, P. Ballone, and R. Jones, Macromolecules 31, 7784 (1998).

${ }^{6}$ B. Montanari, P. Ballone, and R. Jones, Macromolecules 32, 3396 (1999).

${ }^{7}$ C. F. Abrams and K. Kremer, Macromolecules 36, 260 (2003).

${ }^{8}$ M. Dion, H. Rydberg, E. Schröder, D. C. Langreth, and B. I. Lundqvist, Phys. Rev. Lett. 92, 246401 (2004).

${ }^{9}$ M. Dion, H. Rydberg, E. Schröder, D. C. Langreth, and B. I. Lundqvist, Phys. Rev. Lett. 95, 109902(E) (2005).

${ }^{10}$ A. Puzder, M. Dion, and D. C. Langreth, J. Chem. Phys. 124, 164105 (2006).

${ }^{11}$ T. Thonhauser, A. Puzder, and D. C. Langreth, J. Chem. Phys. 124, 164106 (2006).

${ }^{12}$ S. D. Chakarova-Käck, E. Schröder, B. I. Lundqvist, and D. C. Langreth, Phys. Rev. Lett. 96, 146107 (2006).

${ }^{13}$ S. D. Chakarova-Käck, O. Borck, E. Schröder, and B. I. Lundqvist, Phys. Rev. B 74, 155402 (2006).

${ }^{14}$ G. Kresse and J. Furthmüller, Comput. Mater. Sci. 6, 15 (1996).

${ }^{15}$ G. Kresse and J. Furthmüller, Phys. Rev. B 54, 11169 (1996).

${ }^{16}$ J. Perdew, Electronic Structure of Solids '91 (Academic, Verlag, 1991), p. 11.

${ }^{17}$ J. P. Perdew, K. Burke, and Y. Wang, Phys. Rev. B 54, 16533 (1996).

${ }^{18}$ P. E. Blöchl, Phys. Rev. B 50, 17953 (1994).

${ }^{19}$ I. Zanella, A. Fazzio, and A. J. R. da Silva, J. Phys. Chem. B 110, 10849 (2006).

${ }^{20}$ W. A. Hofer, A. J. Fisher, G. P. Lopinski, and R. A. Wolkow,
Phys. Rev. B 63, 085314 (2001).

${ }^{21}$ Y. Jung and M. S. Gordon, J. Am. Chem. Soc. 127, 3131 (2005).

${ }^{22}$ R. Wolkow, G. Lopinski, and D. Moffatt, Surf. Sci. 416, L1107 (1998).

${ }^{23}$ U. Birkenheuer, U. Gutdeutsch, and N. Rösch, Surf. Sci. 409, 213 (1998).

${ }^{24}$ G. Lopinski, D. Moffatt, and R. Wolkow, Chem. Phys. Lett. 282, 305 (1998).

${ }^{25}$ S. Gokhale, P. Trischberger, D. Menzel, W. Widdra, H. Dröge, H.-P. Steinrück, U. Birkenheuer, U. Gutdeutsch, and N. Rösch, J. Chem. Phys. 108, 5554 (1998).

${ }^{26}$ N. Witkowski, F. Hennies, A. Pietzsch, S. Mattsson, A. Föhlisch, W. Wurth, M. Nagasono, and M. N. Piancastelli, Phys. Rev. B 68, 115408 (2003).

${ }^{27}$ N. Witkowski, O. Pluchery, and Y. Borensztein, Phys. Rev. B 72, 075354 (2005).

${ }^{28}$ Y. K. Kim, M. H. Lee, and H. W. Yeom, Phys. Rev. B 71, 115311 (2005).

${ }^{29}$ J.-Y. Lee and J.-H. Cho, Phys. Rev. B 72, 235317 (2005).

${ }^{30}$ M. Mamatkulov, L. Stauffer, C. Minot, and P. Sonnet, Phys. Rev. B 73, 035321 (2006).

${ }^{31}$ J. P. Perdew, K. Burke, and M. Ernzerhof, Phys. Rev. Lett. 77, 3865 (1996).

${ }^{32}$ J. P. Perdew, K. Burke, and M. Ernzerhof, Phys. Rev. Lett. 78, 1396(E) (1997).

${ }^{33}$ J. P. Perdew, K. Burke, A. Zupan, and M. Ernzerhof, J. Chem. Phys. 108, 1522 (1998).

${ }^{34}$ L. Delle Site, C. F. Abrams, A. Alavi, and K. Kremer, Phys. Rev. Lett. 89, 156103 (2002).

${ }^{35}$ C. F. Abrams, L. D. Site, and K. Kremer, Phys. Rev. E 67, 021807 (2003).

${ }^{36}$ J. B. Neaton, M. S. Hybertsen, and S. G. Louie, Phys. Rev. Lett. 97, 216405 (2006).

${ }^{37}$ In this system the most convenient definition for a monolayer (ML) corresponds to one benzene molecule per Si dimer. 